\title{
Programmable purification of type-I polarization-entanglement
}

\author{
Simone Cialdi, ${ }^{1,2}$ Davide Brivio, ${ }^{2}$ and Matteo G. A. Paris ${ }^{2, a)}$ \\ ${ }^{1}$ INFN, Sezione di Milano, I-20133 Milano, Italy \\ ${ }^{2}$ Dipartimento di Fisica, Università degli Studi di Milano, I-20133 Milano, Italy
}

(Received 15 February 2010; accepted 1 July 2010; published online 27 July 2010; publisher error corrected 28 July 2010)

\begin{abstract}
We suggest and demonstrate a scheme to compensate spatial and spectral decoherence effects in the generation of polarization entangled states by type-I parametric downconversion. In our device, a programmable spatial light modulator imposes a polarization dependent phase-shift on different spatial sections of the overall downconversion output and this effect is exploited to realize an effective purification technique for polarization entanglement. (C) 2010 American Institute of Physics. [doi:10.1063/1.3467832]
\end{abstract}

Entanglement is a key resource for several quantum communication and quantum computation protocols. ${ }^{1}$ Entangled states of polarization qubit have been realized exploiting spontaneous parametric down conversion (SPDC) in nonlinear crystals ${ }^{2}$ where a pump photon generates a couple of daughter photons called signal and idler. Brightness and purity of SPDC-based entanglement sources are limited by decoherence. Basically, there are two effects that degrade type-I polarization entanglement. The first is related to the source coherence time and the second to the emission angleand frequency-dependent phase of the SPDC mechanism itself. The effect of a limited coherence of the source may be reduced using a temporal precompensation technique ${ }^{3,4}$ whereas entanglement purity is usually recovered by strong filtering in the angular and spectral SPDC distribution (e.g., narrow irises and spectral filters). Recently very bright source has been realized compensating the angular-phase using suitably prepared birefringent crystals. ${ }^{5}$

In this paper, we suggest and demonstrate an emissionangle and emission-frequency compensation technique based on the use of a programmable one-dimensional spatial light modulator (SLM). This is a crystal liquid phase mask $\left(64 \times 10 \mathrm{~mm}^{2}\right)$ divided in 640 horizontal pixels, each wide $d=100 \mu \mathrm{m}$ and with the liquid crystal $10 \mu \mathrm{m}$ deep. The SLM is set on the path of signal and idler at a distance $D$ $=500 \mathrm{~mm}$ from the two generating crystals. Driven by a voltage, the liquid crystal orientation in correspondence of a certain pixel changes. The photons with an horizontal polarization feel an extraordinary index of refraction depending on the orientation, and this introduce a phase-shift between the horizontal and the vertical polarizations. Since each pixel is driven independently, it is possible to introduce a phase function dependent on the position on the SLM, i.e., on the SPDC generation angles. This kind of devices has been already used as amplitude modulators, ${ }^{6}$ as well as diffractive elements to operate on orbital angular momentum ${ }^{7}$ and for phase-modulation at the single-photon level. ${ }^{8}$ The value of our technique is twofold. On the one hand, our filtering of downconverted photons is drastically reduced, not only in the angular distribution but also in frequency. On the other hand, programmability makes it easily adjustable for many implementations.

\footnotetext{
${ }^{a)}$ Electronic mail: matteo.paris@ fisica.unimi.it.
}

In our scheme (see Fig. 1) the polarization entangled state is generated by two adjacent type-I Beta-Barium Borate (BBO) crystals, ${ }^{2,4,9}$ oriented with their optical axes aligned in perpendicular planes and pumped by a $405 \mathrm{~nm} \mathrm{cw}$ laser diode (Newport LQC405-40P). The part of the pump horizontally polarized generates couples of vertically polarized photons in the first crystal, while the vertical pump polarization generates couples horizontally polarized in the second one. A half-wave plate (HWP) is used to set the pump polarization at $45^{\circ}$ in order to balance the probabilities to generate vertical or horizontal couples of photons.

Taking care of the coherent spatial overlap of the two emission cones the state outgoing the two crystals may be written as $\left|\Phi\left(\omega_{p}, \omega_{s}, \theta\right)\right\rangle=1 / \sqrt{2}\{|H H\rangle$ $\left.+\exp \left[\imath \varphi\left(\omega_{p}, \omega_{s}, \theta\right)\right]|V V\rangle\right\}$, where $\omega_{p}$ and $\omega_{s}$ are the shift from the pump and signal central frequencies $\omega_{p}^{0}$ and $\omega_{s}^{0}=\omega_{p}^{0} / 2$, and $\theta$ is the shift from the central signal angle $\theta_{0}$. Upon expanding all the contributions to the optical paths to first order we obtain the phase function $\varphi\left(\omega_{p}, \omega_{s}, \theta\right)=\phi_{0}+\alpha L \omega_{p}$ $+\beta L \omega_{s}-\delta L \theta$, where $L$ is the crystal's length and $\phi_{0}$ is a constant term depending on the central frequencies and angles. The phase term $\alpha L \omega_{p}$ accounts for the delay time between horizontal and vertical downconverted photons. The

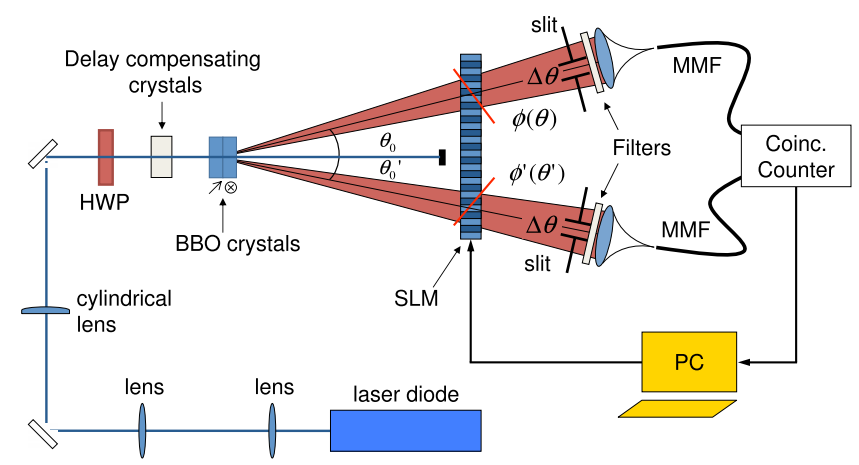

FIG. 1. (Color online) Experimental setup. Polarization entangled states are generated by downconversion in type-I BBO crystals pumped @ 405 nm by a Newport LQC405-40P cw laser diode. Then, a spatial section of the output cones passes through the SLM which provides purification of polarization entanglement. After SLM there are (on both paths) a slit, filters, and a lens that make the image of the pump spot on the crystal into the core of a multimode optical fiber (MMF). For tomographic reconstruction we insert a quarter-wave plate, a HWP, and a polarizer and for the optimization of the phase functions only the polarizers. 

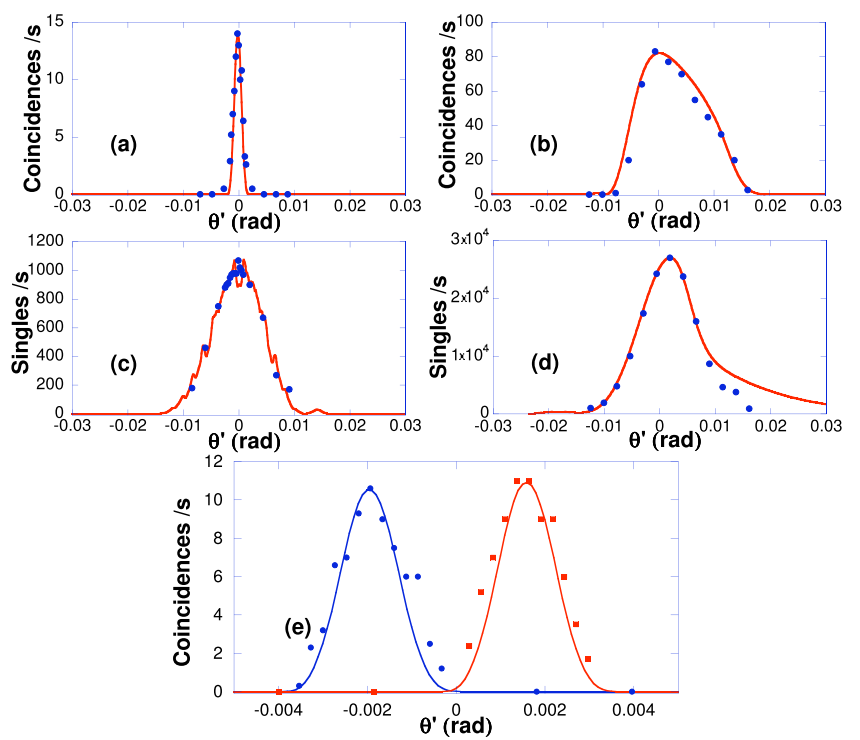

FIG. 2. (Color online) Angular distribution of the idler (around $\theta_{0}^{\prime}=-3^{\circ}$ ) with signal angle set at $\theta_{0}+\theta$. Points are the experimental data; the solid line the theoretical prediction. (a) Coincidences per second with $F_{\mathrm{BP}}, \theta=0$; (b) coincidences per second with $2 \times F_{\mathrm{LP}}, \theta=0$; (c) single counts per second with $F_{\mathrm{BP}}, \theta=0$; (d) single counts per second with $2 \times F_{\mathrm{LP}}, \theta=0$; (e) coincidence per second with $F_{\mathrm{BP}}, \theta= \pm 1.8 \mathrm{mrad}$ (circle/square).

last two terms appear because the photons generated in the first crystal must traverse the second one. These terms may be understood by considering the conservation of the transverse momentum. Taking the signal at the fixed angle $\theta_{0}+\theta$, for different $\omega_{s}$ the idler sweeps different angles $\theta_{0}^{\prime}+\theta^{\prime}$. This means a different optical path and explain the phase term $\beta L \omega_{s}$. Likewise fixing $\omega_{s}$, a positive variation in $\theta$ correspond to a negative variation in $\theta^{\prime}$ and this introduces an optical path dependent on $\theta$, i.e., the phase shift $\delta L \theta$. The conservation of the transverse momentum implies that the signal and idler generation angles $\theta_{0}+\theta$ and $\theta_{0}^{\prime}+\theta^{\prime}$ are not independent. We write $\theta^{\prime}=-\theta+\gamma \omega_{s}+\gamma^{\prime} \omega_{p}$. Within the pump spectral width the dependence on $\omega_{p}$ is negligible and thus we have $\theta^{\prime} \simeq-\theta+\gamma \omega_{s}$, with $\gamma=\partial \theta^{\prime} / \partial \omega_{s}=-0.045$ $\times 10^{-15} \mathrm{rad} / \mathrm{Hz}=1.294 \times 10^{-4} \mathrm{rad} / \mathrm{nm}$. Using this relation and $\delta=2 \beta / \gamma$, which can be proved analytically, the phase function may be written as $\varphi\left(\omega_{p}, \theta, \theta^{\prime}\right)=\phi_{0}+\alpha L \omega_{p}-\beta / \gamma L \theta$ $+\beta / \gamma L \theta^{\prime}$. We exploit the SLM to add the phase functions $\phi(\theta)$ and $\phi^{\prime}\left(\theta^{\prime}\right)$ to the signal and the idler, respectively. This introduces a polarization dependent shift on the $|H H\rangle$ component of $\left|\Phi\left(\omega_{p}, \omega_{s}, \theta\right)\right\rangle$ which, together with temporal delay compensation on the pump, allows one to achieve complete purification. The overall output state may be written as

$$
\begin{aligned}
|\Phi\rangle= & \frac{1}{\sqrt{2}} \int_{-\Delta \theta / 2}^{\Delta \theta / 2} d \theta \int_{-\Delta \theta / 2}^{\Delta \theta / 2} d \theta^{\prime} \int d \omega_{p} f\left(\omega_{p}, \theta_{0}+\theta, \theta_{0}^{\prime}+\theta^{\prime}\right) \\
& \times A\left(\omega_{p}\right)\left[e^{\ell \phi(\theta)+l \phi^{\prime}\left(\theta^{\prime}\right)}|H H\rangle+e^{\imath \varphi\left(\omega_{p}, \theta, \theta^{\prime}\right)}|V V\rangle\right],
\end{aligned}
$$

where $\Delta \theta$ is the angular acceptance due to the presence of the slit. $A\left(\omega_{p}\right)$ is the complex amplitude spectrum of the pump laser and $f=\operatorname{sinc}\left(1 / 2 \Delta k_{\|} L\right)$, where $\Delta k_{\|}$ $=\Delta k_{\|}\left[\omega_{p}, \omega_{s}\left(\theta, \theta^{\prime}\right), \theta\right]$ is the spectral/angular amplitude of the downconverted photons. ${ }^{9}$ We stress that first order effects, i.e., frequency- and angle-dependent phase-shifts at the phase mask are negligible due to the small thickness $(=10 \mu \mathrm{m})$ of the mask. This definitely shows the programmable nature of our device.

In order to assess our model and verify the approximation $\theta^{\prime} \simeq-\theta+\gamma \omega_{s}$, we have measured the angular distribution of the horizontal component of the idler upon setting the signal at the fixed angle $\theta_{0}+\theta$. The angular resolution is $\Delta \theta \simeq 1.2 \mathrm{mrad}$, corresponding to a slit aperture of $1 \mathrm{~mm}$. We verify the absence of diffraction effects using a charge-coupled device camera and measure the angular distribution of coincidences and single counts using two set of filters set in front of each detector. We evaluate numerically the distribution of coincidences $P_{k}\left(\theta_{0}, \theta_{0}^{\prime}\right)$ $\propto \int_{\Delta \theta} d \theta \int_{\Delta \theta} d \theta^{\prime} F_{i}^{2}\left|f\left(0, \theta_{0}+\theta, \theta_{0}^{\prime}+\theta^{\prime}\right)\right|^{2}$ and single counts $P_{k}\left(\theta_{0}, \theta_{0}^{\prime}\right) \propto \int_{\Delta \theta \gg 1} d \theta \int_{\Delta \theta} d \theta^{\prime} F_{i}\left|f\left(0, \theta_{0}+\theta, \theta_{0}^{\prime}+\theta^{\prime}\right)\right|^{2}(i=1,2)$ as obtained omitting integration over pump frequencies (it can be shown that $f\left(\omega_{p}, \theta_{0}+\theta, \theta_{0}^{\prime}+\theta^{\prime}\right) \approx f\left(0, \theta_{0}+\theta, \theta_{0}^{\prime}+\theta^{\prime}\right)$ for crystals length $L \leqq 1 \mathrm{~mm}$ ) and using $F_{1}=F_{\mathrm{BP}}$, a $10 \mathrm{~nm}$ bandpass filters and $F_{2}=F_{\mathrm{LP}}^{2} \times Q E$, i.e., a filter built by two $F_{\mathrm{LP}}$ longpass filters (cut-on wavelength $715 \mathrm{~nm}$ ) and the quantum efficiency $Q E$ of each detector (taken from the specification of the photodiode C30921S). In Fig. 2 we compare the experimental results (points) with the theoretical distributions (solid lines): for panels (a), (b), (c), and (d) $\theta_{0}=3^{\circ}$, whereas for panel (e) the signal is set at $\theta_{0} \pm \theta$, with $\theta=1.8 \mathrm{mrad}$. In Figs. 2(a) and 2(e), the experimental data are deconvolved with the function of the slit. ${ }^{10}$ As it is apparent from the plot, we have very good agreement with the theoretical expectations. The mismatch in the right part of panel (d) is due to the finite size of the phase mask.

For the purification procedure, we set the slits at $\Delta \theta$ $\simeq 6.5 \mathrm{mrad}$ and use, on both channels, the two filters $F_{\mathrm{LP}}$. This allows us to collect the downconverted photons within a wide spectrum and angular distribution. To collect as many photons as possible we make the imaging of the pump spot on the crystals $(\simeq 1.5 \mathrm{~mm})$ into the optical fibers core (diameter of $62.5 \mu \mathrm{m}$ ) using the coupler lenses; the coincidence counts are about $100 / \mathrm{s}$, basically due to low quantum efficiencies of our home made detectors $(<10 \%)$. Notice that the angular acceptance $\Delta \theta$ acts as a $100 \mathrm{~nm}$ bandpass spectral filter for the down converted photons. The delay time between the photons may be compensated upon the introduction of a proper combination of birefringent crystals on the pump path, as already demonstrated in Refs. 3 and 4 (see Fig. 1). Upon setting $\phi^{\prime}\left(\theta^{\prime}\right)=\beta / \gamma L \theta^{\prime}+\epsilon^{\prime}$ and $\phi(\theta)=-\beta / \gamma L \theta+\epsilon$, with $\epsilon^{\prime}+\epsilon=\phi_{0}$, it is possible to compensate all the remaining phase terms in $\varphi\left(\omega_{p}, \theta, \theta^{\prime}\right)$ and to achieve complete purification of the state. With this choice,
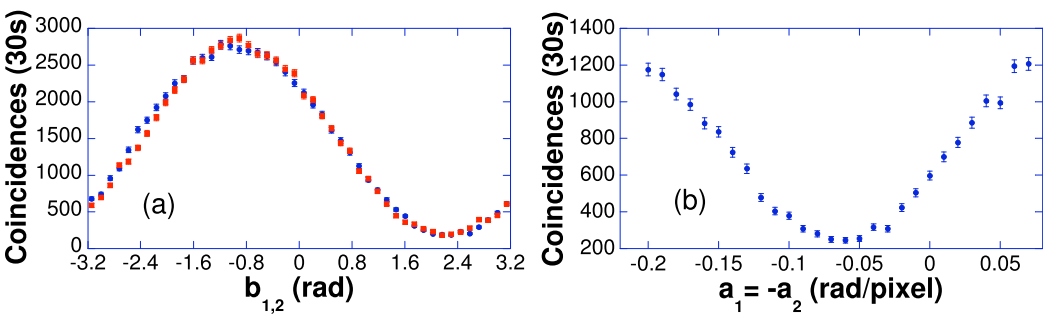

FIG. 3. (Color online) Coincidence counts on a time window of $30 \mathrm{~s}$ with the polarizers in front of the couplers set at $45^{\circ}$ and $-45^{\circ}$. (a) Coincidences as a function of $b_{1,2}$ (circle/square) with $b_{2,1}=0$ and optimal $a_{1,2}$; (b) Coincidences as a function of $a_{1}=-a_{2}$ with optimal $b_{1}$ and $b_{2}=0$. 
we may generate polarization entangled state of the form (1) without any phase term. It is worth noting that the SLM also replaces the birefringent plate used for the optimal generation of photon pairs. ${ }^{2}$ Using the SLM, we set the phase functions $\phi(x)=a_{2}\left(x-x_{c 2}\right)+b_{2}$, and $\phi^{\prime}(x)=a_{1}\left(x-x_{c 1}\right)+b_{1}$, where $x$ is the pixel number, $x-x_{c 2}=(D / d) \theta$ and $x-x_{c 1}=(D / d) \theta^{\prime}$, $x_{c 1}$ and $x_{c 2}$ are the central pixels on idler and signal, i.e., the pixels corresponding to the central angles $\theta_{0}^{\prime}$ and $\theta_{0}$. The values of the parameters $a_{1}, b_{1}, a_{2}$ and $b_{2}$ has been optimized upon inserting two polarizers set at $\alpha_{1}=45^{\circ}$ and $\alpha_{2}=-45^{\circ}$ in front of the couplers and then searching for the minima in the coincidence counts, corresponding to the values of $b_{1,2}$ compensating the constant phase difference $\phi_{0}$ and $a_{1,2}$ removing the angular dependence on $\theta$ and $\theta^{\prime}$. For our configuration $a_{1}=-a_{2}=\beta L d / \gamma D \simeq-0.05, b_{1}+b_{2}=\phi_{0}$.

In Fig. 3(a), we report the coincidence counts on a time window equal to $30 \mathrm{~s}$ as a function of $b_{1}\left(b_{2}\right)$ (blue/red) with $b_{2}=0\left(b_{1}=0\right)$ and with $a_{1,2}$ set to their optimal values. In Fig. 3 (b) we report the coincidence counts on a time window of $30 \mathrm{~s}$ as a function of $a_{1}=-a_{2}$ with $b_{2}=0$ and $b_{1}=\phi_{0}$. The agreement with the theoretical model is excellent. In turn, the purification of the state works as follows: starting from a visibility equal to $0.423 \pm 0.016$, we achieve $0.616 \pm 0.012$ after the delay compensation with the crystals and $0.886 \pm 0.012$ after the spatial compensation with the SLM. The residual lack of visibility is due to low spatial coherence of the pump, which is spatially multimode. Upon adding a constant phase $\zeta$ to the term $b_{1}$ or $b_{2}$ we may also generate a wide zoology of entangled states. With $\zeta=0, \pi$ we have, in ideal conditions, the Bell states $\left|\Phi^{ \pm}\right\rangle$whereas with $\zeta=\pi / 2$ we may generate the state $\left|\Phi_{\pi / 2}\right\rangle=1 / \sqrt{2}[|H H\rangle+\imath|V V\rangle]$.

In order to characterize the output state and to check the effects of the decoherence processes, we have performed state reconstruction by polarization qubit tomography. The procedure goes as follows: we measure a suitable set of independent two-qubit projectors ${ }^{11,12}$ corresponding to different combinations of polarizers and phase-shifters and then the density matrix is reconstructed from the experimental probabilities using maximum-likelihood reconstruction of two-qubit states. The tomographic measurements are obtained by inserting a quarter-wave plate, a HWP and a polarizer. The results are summarized in Fig. 4. Fidelities of the purified polarization states, $|\Phi\rangle=\left|\Phi^{+}\right\rangle,\left|\Phi^{-}\right\rangle$, and $\left|\Phi_{\pi / 2}\right\rangle$, are $F \simeq 0.90 \pm 0.01$, where uncertainty has been evaluated by propagating the errors on the determination of matrix elements by tomography. In order to achieve this precision, we have employed a long acquisition time $(\sim 60 \mathrm{~s})$ thus also demonstrating the overall stability of our scheme. The limited value of the fidelities is basically due to spatial incoherence of the pump and the incomplete temporal delay compensation.
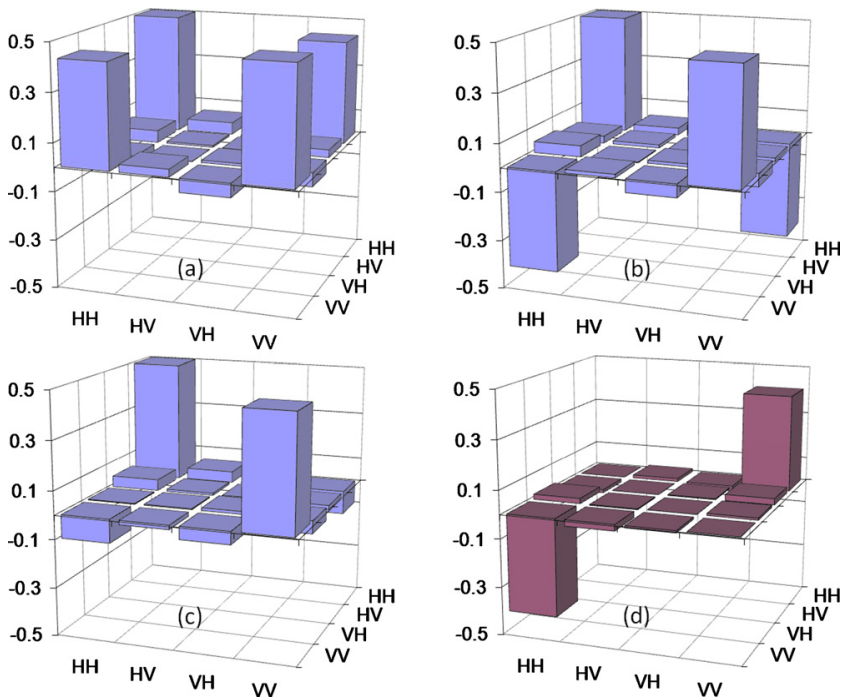

FIG. 4. (Color online) Characterization of the output state. We report the tomographic reconstruction of the purified polarization entangled state (a) $\left|\Phi^{+}\right\rangle\left\langle\Phi^{+}\right|$(real part), (b) $\left|\Phi^{-}\right\rangle\left\langle\Phi^{-}\right|$(real part), (c) real and (d) imaginary part of $\left|\Phi_{\pi / 2}\right\rangle\left\langle\Phi_{\pi / 2}\right|$.

In conclusions, we have suggested and implemented a scheme to compensate spatial and spectral decoherence effects of type-I SPDC-based entangled states. In our device a programmable spatial light modulator acts on different spatial sections of the overall downconversion output and provides polarization entanglement purification. Our device allows the effective generation and purification of type-I polarization entanglement and paves way for quantum information processing with low-cost, low-coherence, and lowpower source of entanglement. ${ }^{13}$

${ }^{1}$ I. L. Chuang and M. A. Nielsen, Quantum Information and Quantum Computation (Cambridge University Press, Cambridge, UK, 2000).

${ }^{2}$ P. G. Kwiat, E. Waks, A. G. White, I. Appelbaum, and P. H. Eberhard, Phys. Rev. A 60, R773 (1999).

${ }^{3}$ Y. Nambu, K. Usami, Y. Tsuda, K. Matsumoto, and K. Nakamura, Phys. Rev. A 66, 033816 (2002).

${ }^{4}$ S. Cialdi, F. Castelli, I. Boscolo, and M. G. A. Paris, Appl. Opt. 47, 1832 (2008).

${ }^{5}$ R. Rangarajan, M. Goggin, and P. Kwiat, Opt. Express 17, 18920 (2009).

${ }^{6}$ G. Lima, A. Vargas, L. Neves, R. Guzman, and C. Saavedra, Opt. Express 17, 10688 (2009).

${ }^{7}$ E. Yao, S. Franke-Arnold, J. Courtial, M. J. Padgett, and S. M. Barnett, Opt. Express 14, 13089 (2006)

${ }^{8}$ S. Cialdi, I. Boscolo, F. Castelli, and V. Petrillo, New J. Phys. 11, 023036 (2009).

${ }^{9}$ S. Cialdi, F. Castelli, and M. G. A. Paris, J. Mod. Opt. 56, 215 (2009).

${ }^{10}$ S. M. Riad, Proc. IEEE 74, 82 (1986).

${ }^{11}$ K. Banaszek, G. M. D'Ariano, M. G. A. Paris, and M. F. Sacchi, Phys. Rev. A 61, 010304(R) (1999).

${ }^{12}$ D. F. V. James, P. G. Kwiat, W. J. Munro, and A. G. White, Phys. Rev. A 64, 052312 (2001).

${ }^{13}$ S. Cialdi, D. Brivio, and M. G. A. Paris, Phys. Rev. A 81, 042322 (2010). 